\title{
The impact of crystalloidal and colloidal infusion preparations on coronary vascular integrity, interstitial oedema and cardiac performance in isolated hearts
}

York A Zausig ${ }^{1 \dagger}$, Daniel Chappell ${ }^{2 \dagger}$, Bernhard F Becker ${ }^{3}$, Daniel Potschka ${ }^{1}$, Hendrik Busse ${ }^{1}$, Kathrin Nixdorf ${ }^{2}$, Diane Bitzinger ${ }^{1}$, Barbara Jacob² and Matthias Jacob ${ }^{2 *}$

\begin{abstract}
Introduction: Recent data suggested an interaction between plasma constituents and the endothelial glycocalyx to be relevant for vascular barrier function. This might be negatively influenced by infusion solutions, depending on ionic composition, $\mathrm{pH}$ and binding properties. The present study evaluated such an influence of current artificial preparations.

Methods: Isolated guinea pig hearts were prepared in a modified Langendorff mode and perfused with KrebsHenseleit buffer augmented with $1 \mathrm{~g} \%$ human albumin. After equilibration the perfusion was switched to replacement of one half buffer by either isotonic saline ( $\mathrm{NaCl}$ ), ringer's acetate (Ri-Ac), 6\% and 10\% hydroxyethyl starch (6\% and 10\% HES, resp.), or $4 \%$ gelatine (Gel), the artificial colloids having been prepared in balanced solution. We analysed glycocalyx shedding, functional integrity of the vascular barrier and heart performance.

Results: While glycocalyx shedding was not observed, diluting albumin concentration towards $0.5 \mathrm{~g} \%$ by artificial solutions was associated with a marked functional breakdown of vascular barrier competence. This effect was biggest with isotonic saline and significantly attenuated with artificial colloids, the difference in the pressure dependent transvascular fluid filtration (basal vs. during infusion in groups $\mathrm{NaCl}, \mathrm{Ri}-\mathrm{Ac}, 6 \% \mathrm{HES}, 10 \% \mathrm{HES}$ and Gel, $n$ $=6$ each) being $0.31 \pm 0.03$ vs. $1.00 \pm 0.04 ; 0.27 \pm 0.03$ vs. $0.81 \pm 0.03 ; 0.29 \pm 0.03$ vs. $0.68 \pm 0.02 ; 0.32 \pm 0.03$ vs. $0.59 \pm 0.08$ and $0.31 \pm 0.04$ vs. $0.61 \pm 0.03 \mathrm{~g} / 5 \mathrm{~min}$, respectively. Heart performance was directly related to $\mathrm{pH}$ value $(7.38 \pm 0.06,7.33 \pm 0.03,7.14 \pm 0.04,7.08 \pm 0.04,7.25 \pm 0.03)$, the change in the rate pressure product being $21,702 \pm 1969$ vs. $21,291 \pm 2,552 ; 22,098 \pm 2,115$ vs. $14,114 \pm 3,386 ; 20,897 \pm 2,083$ vs. $10,671 \pm 1,948 ; 21,822 \pm$ 2,470 vs. $10,047 \pm 2,320$ and $20,955 \pm 2,296$ vs. $15,951 \pm 2,755 \mathrm{mmHg} \times$ bpm, respectively.

Conclusions: It appears important to maintain the $\mathrm{pH}$ value within a physiological range to maintain optimal myocardial contractility. Using colloids prepared in calcium-containing, balanced solutions for volume replacement therapy may attenuate the breakdown of vascular barrier competence in the critically ill.
\end{abstract}

\section{Introduction}

For over 100 years vascular barrier competence was generally acknowledged to be sufficiently explained by the historical principle of Ernest Starling [1]. This stipulated an inwardly directed oncotic gradient between an interstitial

\footnotetext{
* Correspondence: matthias.jacob@med.uni-muenchen.de

+ Contributed equally

${ }^{2}$ Department of Anaesthesiology, University Hospital Munich, Nussbaumstr.

20, 80336 Munich, Germany

Full list of author information is available at the end of the article
}

space presumably low in protein and the protein rich plasma with percentage albumin at concentrations of around $4 \mathrm{~g} \%$ as main constituent. This gradient was considered to keep the compartments in balance, despite an intravascular hydrostatic pressure, which forces fluids and solutes outwards. The therapeutical target to prevent interstitial oedema whilst maintaining cardiac preload in the face of an unaffected endothelial cell line would have been, according to this model, to merely maintain the

\section{Ciomed Central}


oncotic plasma pressure. The practical answer was the intravenous infusion of isooncotic colloids prepared in isotonic saline, irrespective of their binding properties for electrolytes and membrane coatings.

The last decade, however, has brought increasing evidence that things might not be that easy. Various experimental models showed that the interstitial oncotic pressure in most organs is far from zero and, surprisingly, does not relevantly influence transvascular filtration behaviour [2]. This brought attention to the endothelial glycocalyx, a negatively charged layer of proteoglycans and glycosaminoglycans, now identified as an important part of vascular barrier competence [3]. Due to its special biophysical and biochemical properties, the glycocalyx binds plasma constituents, forming the endothelial surface layer with a functional thickness of more than $1 \mu \mathrm{m}$ [4]. It is only the oncotic gradient across this layer, that is, between the protein-loaded glycocalyx and a small space low in protein directly beneath, but completely at the luminal side of the anatomical vessel wall, that helps to limit hydrostatically driven outflow of plasma constituents in high-pressure segments of the circulation [2]. In addition, the endothelial surface layer plays an important role for shear stress transduction to the endothelial cells and also for generating an anti-inflammatory, antithrombotic and anti-adhesive vascular surface by harbouring adhesion molecules [5]. The fragile glycocalyx, however, can be degraded in various pathophysiological situations such as ischaemia/reperfusion, sepsis, hyperglycaemia, trauma or diabetes $[6,7]$.

The integrity of the endothelial surface layer, which seems to be strongly related to oedema formation and cardiac performance [6], is strongly dependent on sufficient concentrations of suitable plasma constituents [8]. Moreover, the binding properties of both glycocalyx and plasma proteins should depend on plasma $\mathrm{pH}$. Thus, acid-base chemistry should be considered when clinicians assess whether the composition of infusion preparations is adequate. Furthermore, some constituents of these preparations might disrupt the competence of the endothelial surface layer.

The present study investigated the influence of commercially available crystalloidal and colloidal infusion preparations on coronary vascular integrity, interstitial oedema and cardiac performance of isolated guinea-pig hearts. It extends the insight into what actually happens to microcirculation when diluting natural plasma constituents with artificial substitutes beyond a critical border. Therefore, these data might already help us today to rationally select a substitution solution for a critically ill patient, even in the absence of outcome-based evidence. Beyond that, such basic data may help to sharpen the focus of future developments in the field.

\section{Materials and methods}

The investigation conformed to the Guide for the Care and Use of Laboratory Animals published by the US National Institutes of Health (NIH Publication No. 85-23, revised 1996) and was approved by - and licensure of the investigator obtained from - the Government of Upper Bavaria (Regierung von Oberbayern file No. 209.1/2112531.3-3/99).

Hearts were prepared in a non-working Langendorff mode comparing basal conditions to intracoronary infusion of different artificial solutions (details see below). The unblinded study was arranged in three steps. Protocol 1 (Figure 1) assessed transvascular filtration immediately before and during infusion of the respective test solution, as well as interstitial water content at the end of protocol. Also, quantitative and qualitative glycocalyx shedding was determined. According to protocol 2 (Figure 1), human polymorphonuclear neutrophilic granulocytes (PMN) were infused intracoronarily and their adhesion rate was assessed. Protocol 3 (Figure 1) compared changes of heart performance and ionic and $\mathrm{pH}$ compositions when infusing the different solutions. The normal ranges for electrolytes, bicarbonate, phosphate and lactate in human plasma, in comparison to the composition of the tested crystalloidal and colloidal solutions, are presented in Table 1.

\section{Heart preparation and perfusion}

\section{Basal preparations (protocols 1 and 2) (Figure 1)}

Guinea pigs (male; weight 300 to $400 \mathrm{~g}$ ) were stunned by neck dislocation, and immediately after opening of the thorax had their hearts arrested with ice-cold isotonic saline. The aorta was cannulated and the coronary arteries were reperfused in situ at a constant flow rate of $6 \mathrm{ml} / \mathrm{min}$ ute (mean $37 \pm \mathrm{SD} 0.5^{\circ} \mathrm{C}, \mathrm{pH} 7.40 \pm 0.05$ ). The perfusate was administered by a peristaltic pump (Type: MS-1 REGLO/8-160; Ismatec ${ }^{\mathrm{TM}}$ SA Laboratoriumstechnik, Glattbrugg-Zürich, Switzerland), the perfusion pressure being continuously recorded with a pressure transducer (FMI $\mathrm{GmbH}$, Engelsbach, Germany). An initial perfusion pressure above $80 \mathrm{~cm} \mathrm{H}_{2} \mathrm{O}$ predicted heart failure and led to exclusion. Hearts were removed from the thorax and preparation was finalised by ligating the caval and azygos veins and inserting a cannula into the pulmonary artery $[9,10]$.

\section{Additional preparations (protocol 3) (Figure 1)}

A thin, saline-filled latex balloon (Hugo Sachs Electronic KG, March-Hugstetten, Germany) was inserted into the left ventricle and attached via a metal cannula to a pressure transducer (Isotec, Hugo Sachs Electronic KG) enabling measurement of isovolumetric systolic left ventricular pressure (LVP) development.

The initial intracoronary perfusion was carried out using a modified Krebs-Henseleit buffer (KHB, composition: $116 \mathrm{mM} \mathrm{NaCl}, 23 \mathrm{mM} \mathrm{NaHCO}$, $3.6 \mathrm{mM} \mathrm{KCl}, 1.16 \mathrm{mM}$ 


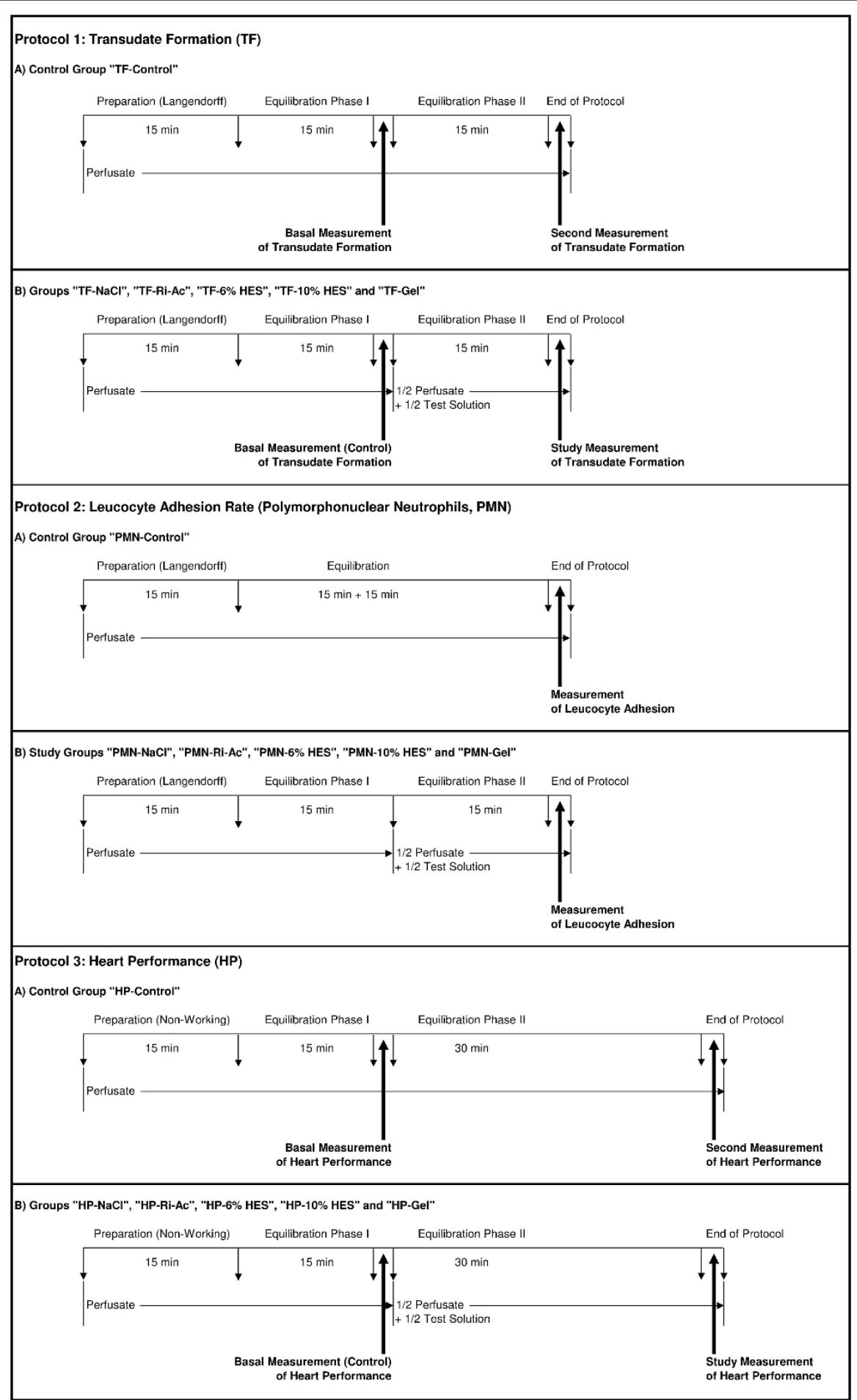

Figure 1 Experimental protocols. Protocol 1: transudate formation (TF). Sample generation for assessing transudate formation and glycocalyx shedding in control (TF-Control) and study groups (TF-NaCl, TF-Ri-Ac, TF-6\% HES, TF-10\% HES, and TF-Gel). The same hearts were used to determine interstitial water content after the protocol, and in two additional cases in each group, for electron microscopy evaluation. Protocol 2: polymorphonuclear neutrophils (PMN). The intracoronary adhesion rates of PMN were determined in control (PMN-Control) and study groups (PMN$\mathrm{NaCl}, \mathrm{PMN}-\mathrm{Ri}-\mathrm{Ac}, \mathrm{PMN}-6 \% \mathrm{HES}, \mathrm{PMN}-10 \%$ HES, and PMN-Gel) at the time indicated. Application and evaluation followed the detailed description given within the running text. Protocol 3: heart performance (HP). The determination of heart performance occurred in control (HP-Control) and study groups (HP-NaCl, HP-Ri-Ac, HP-6\% HES, HP-10\% HES, and HP-Gel) at the times indicated. For details, see Materials and methods. 
Table 1 Normal ranges of electrolyte, bicarbonate, phosphate and lactate in comparison to the composition of the studied solutions

\begin{tabular}{|c|c|c|c|c|c|c|c|}
\hline & Plasma & $\mathrm{NaCl}$ & $\mathrm{Ri}-\mathrm{Ac}$ & $6 \%$ HES & $10 \% \mathrm{HES}$ & Gel & Unit of measurement \\
\hline \multicolumn{8}{|l|}{ Kations } \\
\hline Sodium & 137 to 147 & 154 & 130 & 130 & 130 & 144 & $\mathrm{mmol} / \mathrm{l}$ \\
\hline Potassium & 3.5 to 5.5 & NA & .5 .4 & .5 .5 & .5 .5 & 5 & $\mathrm{mmol} / \mathrm{l}$ \\
\hline Magnesium & 0.8 to 1.0 & NA & 1 & 1 & 1 & .1 .5 & $\mathrm{mmol} / \mathrm{l}$ \\
\hline Calcium & 2.2 to 2.6 & NA & 0.9 & 1 & 1 & .2 .5 & $\mathrm{mmol} / \mathrm{l}$ \\
\hline \multicolumn{8}{|l|}{ Anions } \\
\hline Chloride & 95 to 110 & 154 & 112 & 112.5 & 112.5 & 103 & $\mathrm{mmol} / \mathrm{l}$ \\
\hline Bicarbonate & 23 to 25 & NA & NA & NA & NA & NA & $\mathrm{mmol} / \mathrm{l}$ \\
\hline Phosphate & 0.8 to 1.6 & NA & NA & NA & NA & NA & $\mathrm{mmol} / \mathrm{l}$ \\
\hline Acetate & NA & NA & 27 & 27 & 27 & 27 & $\mathrm{mmol} / \mathrm{l}$ \\
\hline Lactate & 0.0 to 1.0 & NA & NA & NA & NA & NA & $\mathrm{mmol} / \mathrm{l}$ \\
\hline \multicolumn{8}{|l|}{ Colloids } \\
\hline HES & NA & NA & NA & 6 & 10 & NA & $g \%$ \\
\hline Gelatine & NA & NA & NA & NA & NA & 4 & $g \%$ \\
\hline Theoretical osmolality & 280 to 300 & 308 & 276 & 277 & 277 & 283 & mosmol/kg H $\mathrm{H}_{2} \mathrm{O}$ \\
\hline
\end{tabular}

$\mathrm{NaCl}$, isotonic saline; Ri-Ac, ringer's acetate; $6 \%$ and 10\% HES: $6 \%$ and 10\% hydroxyethyl starch in Ringer's acetate. NA (not applicable).

$\mathrm{KH}_{2} \mathrm{PO}_{4}, 1.25 \mathrm{mM} \mathrm{CaCl}_{2}, 0.58 \mathrm{mM} \mathrm{MgSO}_{4}, 5.4 \mathrm{mM}$ glucose, $0.3 \mathrm{mM}$ pyruvate, and $2.8 \mathrm{U} / 1$ insulin, gassed with 94.5\% $\mathrm{O}_{2}$ and 5.5\% $\mathrm{CO}_{2}$ ) augmented with $1 \mathrm{~g} \%$ human albumin from the onset of preparation. Electrolytes, $\mathrm{pH}$ and oxygen tension (Gem Premier 4000, Il GmbH, Kirchheim, Germany) and osmolality (Advanced Osmometer Model 2000, Advanced Instruments, Norwood, MA, USA) were measured.

\section{Experimental protocols}

All hearts were randomly assigned to the respective groups before starting the preparation.

\section{Protocol 1 (Figure 1): transudate formation, glycocalyx shedding and interstitial water content (TF) Transudate formation}

Transudate filtered from the coronary system was collected on the epicardial surface in timed aliquots and quantified while the coronary venous effluent was collected from the pulmonary artery. Following preparation and the first equilibration phase in a dry warming chamber, the basal measurement of transudate formation was performed between minutes 25 and 30 . In the control group (TF-Control, $n=6$, protocol $1 \mathrm{~A}$ ) the perfusion continued for another 15 minutes before a second collection was carried out (minutes 45 to 50 ). In the study groups (TF-NaCl, TF-Ri-Ac, TF-6\% HES, TF-10\% HES and TF-Gel, $n=6$ each, protocol $1 \mathrm{~B}$ ), the perfusion was switched after 15 minutes to a 1:1 mixture of buffer and the respective test solution following the basal measurement of transudate formation. The final determination was performed between minutes 30 and 45 following a second equilibration interval.

\section{Glycocalyx shedding}

Samples of coronary effluent were obtained from the same hearts after the first equilibration phase and again at the end of the second equilibration phase, each time for 3 minutes, and were stored at $-20^{\circ} \mathrm{C}$. The concentration of syndecan-1 (CD 138), a main component of the glycocalyx, was determined using an enzyme-linked immunosorbent assay (Diaclone Research, Besancon, France) as described before [11].

Tissue water content

At the end of the protocol, oedema formation was assessed in each heart by measuring ventricular wet weight (at once) and dry weight (after $24 \mathrm{~h}$ at $60^{\circ} \mathrm{C}$ ). With interstitial oedema formation the quotient - wet weight $\times$ dry weight $^{-1}$ - increases in vivo from a mean value of 4.76 [8].

Electron microscopy

Two additional hearts per group were treated as described in protocol 1 for the TF-Control, TF- NaCl, TF-Ri-Ac, TF$6 \%$ HES, TF-10\% HES and TF-Gel groups, but were prepared for electron microscopy (EM) as described previously [4] to qualitatively screen for morphological correlates of infusion-related glycocalyx shedding.

\section{Protocol 2 (Figure 1): adhesion of polymorphonuclear neutrophils}

Following preparation and the first equilibration phase the Langendorff-perfusion continued for another 15 minutes in the control group (PMN-Control, $n=6$, protocol 2A) before application of PMN. In the study groups (PMN$\mathrm{NaCl}, \mathrm{PMN}-\mathrm{Ri}-\mathrm{Ac}, \mathrm{PMN}-6 \% \mathrm{HES}, \mathrm{PMN}-10 \%$ HES and PMN-Gel, $n=6$ each, protocol 2B), the perfusion mode switched to a 1:1 mixture of buffer and the respective test solution after the first equilibration interval. A second 
interval between minutes 30 and 45 preceded the assessment of PMN adhesion under the respective study conditions.

PMN of guinea pigs and humans show a quantitatively similar degree of adhesion in our model [12]. Therefore, the latter were used to substantially reduce the total number of animals needed. Preparation, intracoronary application and counting of non-adherent human PMN, as well as calculation of their adhesion rate were carried out as described previously [12]. Under basal conditions intracoronary retention has been shown to be $21.6 \pm$ $2.1 \%$ [12]. Experimental shedding of the glycocalyx by intracoronary application of heparinase almost doubled this adhesion rate to $37.3 \pm 2.0 \%[4,13]$.

\section{Protocol 3: heart performance (HP) (Figure 1)}

For assessment of ventricular pressure development, balloon volume was adjusted to an initial diastolic LVP of 0 $\mathrm{mmHg}$ during the control period. Thus, any increase in diastolic LVP reflected an increase in left ventricular wall stiffness or diastolic contracture. The rate pressure product (RPP) was calculated as follows [14]:

$\mathrm{RPP}=($ Left ventricular systolic pressure - Left ventricular diastolic pressure $) \times \mathrm{HR}$

Following preparation and equilibration phase I, baseline values of HR, LVP and RPP were obtained. After that, basal perfusion was continued (HP-Control, $n=6$, protocol 3A) or switched to the 1:1 mixture of buffer and the respective test solution (HP-NaCl, HP-Ri-Ac, HP-6\% HES, HP-10\% HES and HP-Gel, $n=6$ each, protocol 3B). $\mathrm{HP}$ was re-assessed following equilibration phase II.

\section{Statistical analysis}

The measured data are presented as mean \pm SD, with $n$ indicating the number of experiments.

To calculate the number of hearts needed, we performed a power calculation with G*Power 3.1 according to Faul et al. [15]. With $n=6$, alpha-level of 0.05 and an assumed effect size

$d>1$, we expected a power level of $95 \%$ for the pairwise $t$-test with matched pairs. This number would also fit analyses of variance (ANOVA) with six groups and comparable values. The data for the effect size calculation being more than 1SD were based on previous studies [8]. Comparisons were made using the Student $t$-test for paired samples. For multiple comparisons, ANOVA was performed with the Bonferroni correction. In the case of unequal variances (group TF-Control and TF- $\mathrm{NaCl}$ ), identified by the Levene test for homogeneity of variances, Dunnett's T3-test was performed. A P-value less than 0.05 was considered significant. The statistical software used to conduct the analyses was SPSS 16 (SPSS Inc., Chicago, IL, USA).

\section{Results}

Electron microscopy of exemplary hearts from the control and the 6\% HES group showed a tight glycocalyx at the endothelial surface at the end of perfusion protocols (Figure 2). Because no morphological differences were evident, tissue of hearts from other groups was not further investigated at this stage.

Transudate formation and parameters related to HP showed no significant intergroup differences under basal conditions or between basal and second measurements in the control groups, respectively. However, a significant increase in transudate formation versus control conditions was observed in all study groups upon dilution of the modified, albumin-augmented KHB by artificial solutions (Figure 3). Vascular leakage was highest in the colloid-free groups TF- $\mathrm{NaCl}$ and TF-Ri-Ac, but significantly attenuated versus that in colloid groups TF-6\% HES, TF-10\% HES and TF-Gel. A complementary picture was found for the tissue water content, the wet-to-dry weight ratio for groups TF-NaCl, TF-Ri-Ac, TF-6\% HES, TF-10\% HES and TF-Gel $(7.29 \pm 0.03,7.16 \pm 0.36,7.00 \pm 0.21,6.83 \pm 0.09$ and $6.45 \pm 0.21$, respectively) being significantly increased versus TF-Control $(6.01 \pm 0.03 ; n=6$ for each group; TF-Control versus TF-NaCl, TF-Ri-Ac, TF-6\% HES and TF-10\% HES: $P<0.05$; TF-10\% HES versus TF-Control and TF-NaCl: $P<0.05$; TF-Gel versus TF-Control, TF- $\mathrm{NaCl}$ and TF-6\% HES: $P<0.05$; TF- $\mathrm{NaCl}$ versus TF-Control, TF- $10 \%$ HES and TF-Gel: $P<0.05$; ANOVA: $\mathrm{df}=5 ; F=25.380 ; P=0.000$; pairwise post hoc testing performed with Dunnett's T3-test).

PMN adhesion rates of groups PMN-Control, PMN$\mathrm{NaCl}, \mathrm{PMN}-\mathrm{Ri}-\mathrm{Ac}, \mathrm{PMN}-6 \% \mathrm{HES}, \mathrm{PMN}-10 \%$ HES and PMN-Gel were $20.3 \pm 0.6,21.5 \pm 0.5,20.5 \pm 1.0,21.7 \pm$ $1.2,21.9 \pm 1.1$ and $20.6 \pm 0.8 \%$, respectively $(n=6$ for each group). Though adhesion of PMN during infusion of $10 \%$ hydroxyethyl starch was significantly elevated versus control conditions (ANOVA: $\mathrm{df}=5 ; F=3.777$; $P=0.009$; pairwise post hoc test with Bonferroni correction: PNM-10\% HES versus PNM-Control: $P=0.04$; all others not significant), this effect was minimal. Shedding of syndecan-1 did not significantly differ among any of the investigated groups (not shown).

Substituting one half of the KHB by 6\% HES (HP-6\% HES), 10\% HES (HP-10\% HES) and HP-Ri-Ac decreased heart rate significantly compared with the respective basal value $(174 \pm 18$ versus $221 \pm 14,176 \pm 22$ versus $216 \pm 19$ and $198 \pm 25$ versus $222 \pm 17 \mathrm{bpm}$ ), whereas no significant change was observed in group HP-Gel (222 \pm 32 versus $217 \pm 21 \mathrm{bpm})$. $\mathrm{HP}-\mathrm{NaCl}$, by contrast, showed a significant increase compared to the basal value $(249 \pm$ 14 versus $220 \pm 21 \mathrm{bpm} ; n=6$ for each group; respective control versus study measurement of $\mathrm{HP}-\mathrm{NaCl}, \mathrm{HP}-\mathrm{Ri}$ Ac, HP-6\% HES and HP-10\% HES: $P<0.05$; $\mathrm{HP}-\mathrm{NaCl}$ 

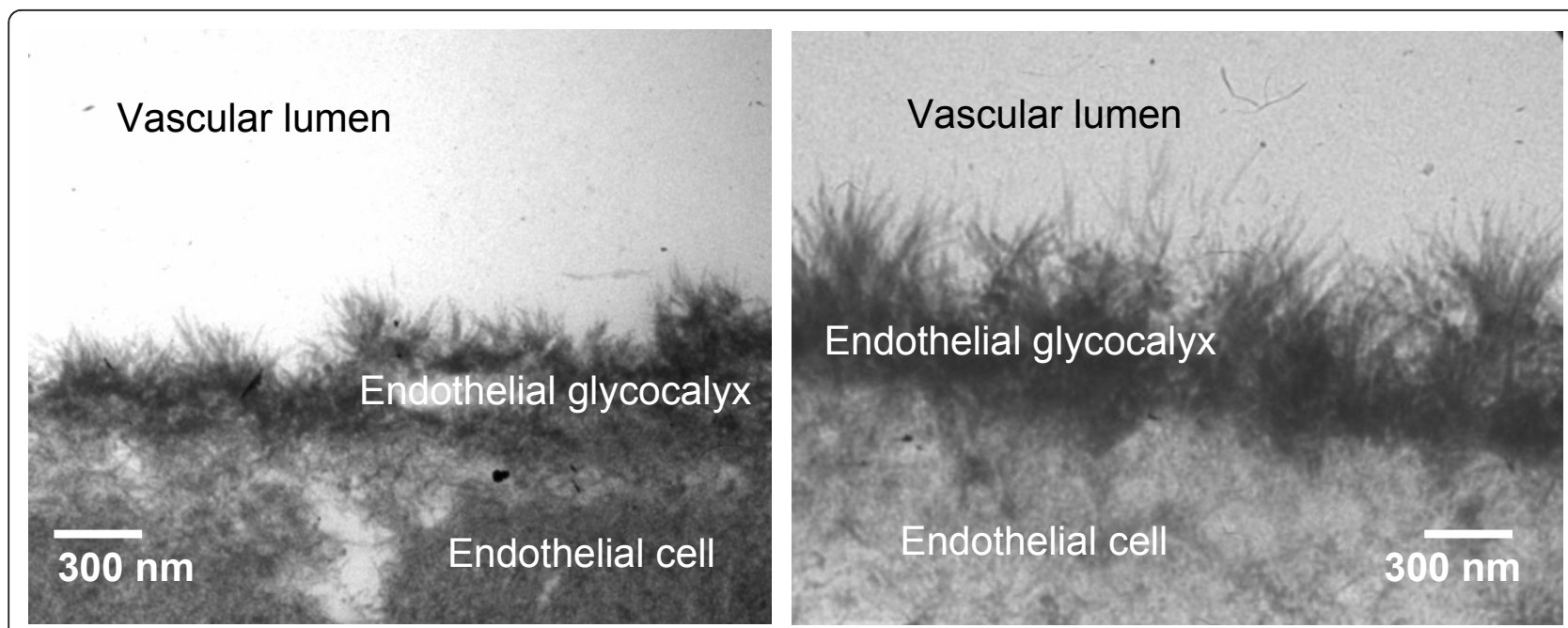

Figure 2 Endothelial glycocalyx. Exemplary electron microscopy picture of an intact endothelial glycocalyx after perfusion under control conditions (TF-Control, left panel) and after intracoronary infusion of $6 \%$ hydroxyethyl starch (TF-6\% HES, right panel).

and HP-Gel versus HP-6\% HES and HP-10\% HES: $P<0.05$; ANOVA: $\mathrm{df}=5 ; \mathrm{F}=19.458 ; P<0.001$; pairwise post hoc testing performed with the Bonferroni correction).

LVP remained constant in control hearts $(94 \pm 12$ versus $96 \pm 11 \mathrm{mmHg}$ ), but decreased significantly during perfusion in all study groups versus the respective basal value $(71 \pm 13$ versus $100 \pm 9 ; 61 \pm 7$ versus $95 \pm 11 ; 57 \pm 11$ versus $101 \pm 10$ and $72 \pm 12$ versus $97 \pm 6 \mathrm{mmHg}$ for groups HP-Ri-Ac, HP-6\% HES, HP-10\% HES and HP-Gel, respectively), except for $\mathrm{HP}-\mathrm{NaCl}(86 \pm 14$ versus $99 \pm$ $8 \mathrm{mmHg} ; n=6$ for each group; control versus HP-Ri-Ac, HP-6\% HES, HP-10\% HES and HP-Gel: $P<0.05 ; \mathrm{HP}-\mathrm{NaCl}$ versus HP-10\% HES: $P<0.05$; ANOVA: $\mathrm{df}=5 ; F=11.364$; $P<0.001$; pairwise post hoc testing performed with the Bonferroni correction).

These alterations resulted in significant decreases in the RPP compared to basal values $(14,114 \pm 3,386$ versus $22,098 \pm 2,115 ; 10,671 \pm 1,948$ versus $20,897 \pm 2,083$;

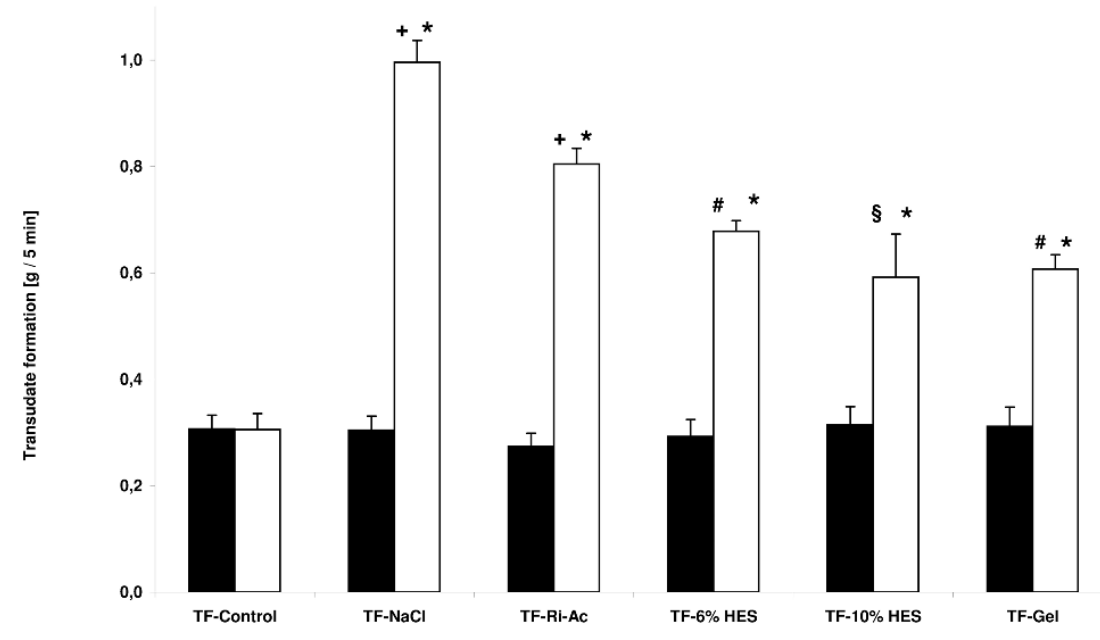

Figure 3 Vascular integrity. Transudate formation (all data expressed as mean $\pm S D ; n=6$ for each group). Filled bars, basal measurement; open bars, second or study measurement. There were no significant differences among the basal measurements of all groups (analysis of variance (ANOVA): $\mathrm{df}=5 ; F=0.444 ; P=0.814$ ). ${ }^{*} P<0.05$ basal measurement versus study measurement (Student's paired $t$-test); ${ }^{+} P<0.05$ versus all other basal and second/study measurements; ${ }^{~} P<0.05$ versus all other basal and second/study measurements except study measurement of TF-10\% HES; ${ }^{\S} P<0.05$ versus all other basal and second/study measurements except TF-6\% HES and TF-Gel (ANOVA: df $=5 ; F=172,644$; $P=0.000$; pairwise post hoc test performed with Dunnett's T3-test). 
$10,047 \pm 2,320$ versus $21,822 \pm 2,470$ and $15,951 \pm 2,755$ versus 20,955 $\pm 2,296 \mathrm{mmHg} \times \mathrm{bpm}$ ) (Figure 4).

The perfusate $\mathrm{pH}$ value was significantly reduced versus the modified KHB during perfusion with Ri-Ac (7.33 \pm $0.03), 6 \%$ HES (7.14 \pm 0.04$), 10 \%$ HES $(7.08 \pm 0.04)$ and Gel (7.25 \pm 0.03$)$, all $P<0.05$, whereas $\mathrm{NaCl}$ did not cause a significant change (7.38 $\pm 0.06 ; P>0.05)$. During infusion of artificial solutions, electrolyte concentrations in the aortic inflow showed drug-dependent deviations. There was a reduction of sodium and calcium in all study groups with the exception of the $\mathrm{NaCl}$ group (Table 2). Potassium was only significantly decreased versus the basal perfusate in the $\mathrm{NaCl}$ group, whereas all other solutions led to a slight, albeit significant increase $(P<0.05)$. Osmolality was not different among the groups (Table 2).

\section{Discussion}

Our study evaluated the impact of intracoronary infusion of commercially available crystalloidal and colloidal infusion preparations (test solutions, see Table 1) on coronary vascular integrity, HP, PMN adhesion and oedema formation of isolated guinea-pig hearts, with special focus on the endothelial glycocalyx. According to recent knowledge we augmented the basal Krebs-Henseleit perfusate with $1 \mathrm{~g} \%$ human albumin to generate a sufficiently functional endothelial surface layer. As in vivo, this results from combining an intact endothelial glycocalyx with plasma proteins. Previous work $[4,8]$ and preliminary experiments suggested that it might be difficult to generate marked disturbances of the endothelial surface layer when starting with physiological albumin concentrations around
$4 \mathrm{~g} \%$, because coronary washout requires too long [8]. Accordingly, by augmenting the buffer with merely $1 \mathrm{~g} \%$ human albumin we simulated conditions of a hypo-albuminaemic patient. Infusion of artificial solutions was simulated by replacing one half of the initial buffer, leading to a further reduction of the intracoronary albumin content towards $0.5 \mathrm{~g} \%$. This protocol disclosed some marked effects.

Actual shedding of the endothelial glycocalyx was obviously not initiated by any solution or condition simulated, as demonstrated by no increase in the glycocalyx constituent syndecan-1 in coronary effluent, no morphological hint on the basis of electron microscopic examination, and no relevant increase in leukocyte adhesion. However there were some considerable functional defects of vascular competence. In particular, we observed an increase in transudate, the equivalent of lymph flow in the isolated heart model and a direct measure of vascular fluid leak. This was noted especially when using colloidfree infusion solutions, these obviously diluting the colloid content of the net perfusate below a critical level. Presumably, this pathological microvascular disturbance is reversible, because no overt damage to the glycocalyx was detected.

Interestingly, infusion of the non-balanced sodium chloride had, despite a physiological pH-value of the mixed perfusate, a significantly worse effect on vascular leak than infusion of the balanced Ringer's acetate solution, which led to a slight metabolic acidosis of the perfusate in our model. These effects on $\mathrm{pH}$ value, however, are totally contrary to those seen under clinical conditions, where the

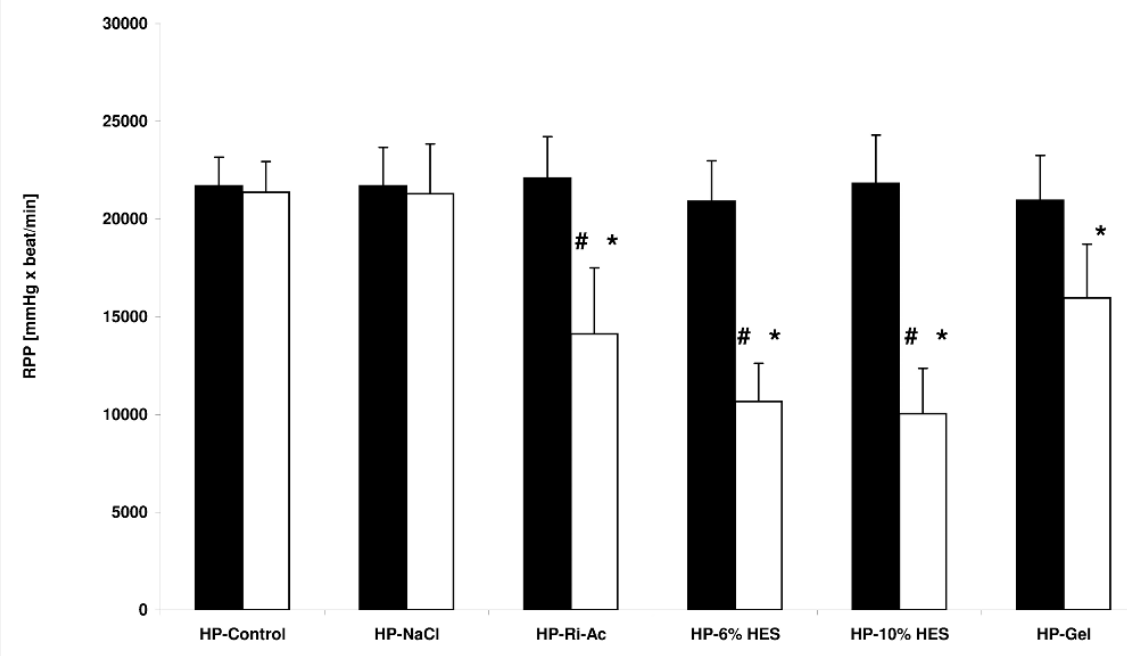

Figure 4 Haemodynamic performance. Rate-pressure product (RPP, all data expressed as mean \pm SD; $n=6$ for each group). Filled bars, basal measurement; open bars, second or study measurement. ${ }^{*} P<0.05$ versus Control; ${ }^{\#} P<0.05$ versus $\mathrm{HP}-\mathrm{NaCl}$ (analysis of variance: $\mathrm{df}=5$; $F=29.361 ; P<0.001$; pairwise post hoc testing performed with the Bonferroni correction). 
Table 2 Values measured within effective perfusate during study phase without (HP-Control) and with (HP-NaCl, HP-Ri-Ac, HP-6\% HES, HP-10\% HES and HP-Gel) infusion of respective preparation according to the study protocol

\begin{tabular}{rlllllll}
\hline & HP-Control & HP-NaCl & HP-Ri-Ac & HP-6\% HES & HP-10\% HES & HP-Gel & Unit of measurement \\
\hline Sodium & $139 \pm 1$ & $148 \pm 2^{*} \#$ & $133 \pm 2^{*}$ & $134 \pm 4^{*}$ & $136 \pm 2^{*}$ & $136 \pm 2^{*}$ & $\mathrm{mmol} / \mathrm{l}$ \\
Potassium & $.3 .78 \pm 0.04$ & $.1 .77 \pm .0 .05^{*} \#$ & $.4 .41 \pm .0 .09^{*}$ & $.4 .46 \pm .0 .12^{*}$ & $.4 .49 \pm .0 .09^{*}$ & $.4 .21 \pm .0 .09^{*}$ & $\mathrm{mmol} / \mathrm{l}$ \\
Calcium & $.1 .86 \pm 0.05$ & $.0 .97 \pm .0 .04^{*} \#$ & $.1 .33 \pm .0 .02^{*}$ & $.1 .39 \pm .0 .05^{*}$ & $.1 .44 \pm .0 .04^{*}$ & $.1 .49 \pm .0 .11^{*}$ & $\mathrm{mmol} / \mathrm{l}$ \\
Osmolarity & $269 \pm 26$ & $266 \pm 34$ & $256 \pm 24$ & $274 \pm 16$ & $276 \pm 25$ & $276 \pm 5$ & $\mathrm{mosmol} / \mathrm{l}$ \\
pH & $.7 .43 \pm 0.04$ & $.7 .38 \pm .0 .06$ & $.7 .33 \pm .0 .03^{*}$ & $.7 .14 \pm .0 .04^{*}$ & $.7 .08 \pm .0 .04^{*}$ & $.7 .25 \pm .0 .03^{*}$ & \\
\hline
\end{tabular}

Electrolyte concentrations, osmolarity and $\mathrm{pH}$ in perfusate, assessed in the delivering lines of the HP groups under basal conditions (HP-Control) and after having switched to infusion mode using the respective study solutions (HP-NaCl, HP-Ri-Ac, HP-6\% HES, HP-10\% HES, and HP-Gel). All data expressed in mean \pm SD; $n=$ 6 for each group; ${ }^{*} P<0.001$ versus HP-Control; ${ }^{\# P}<0.001$ versus HP-Ri-Ac, HP-6\% HES, HP-10\% HES, and HP-Gel. HP, heart performance; NaCl, isotonic saline; RiAc, ringer's acetate; $6 \%$ and $10 \%$ HES: $6 \%$ and $10 \%$ hydroxyethyl starch in Ringer's acetate.

infusion of sodium chloride is associated with severe hyperchloraemic acidosis, which does not occur with balanced solutions [16]. The reason is that after intravenous infusion into the human circulation, the anion acetate is rapidly metabolised into bicarbonate, preventing acidosis. The isolated heart is obviously unable to metabolise acetate rapidly enough to prevent an artificial acidosis. Therefore, the antiacid effect of balanced solutions might be underestimated in this in-vitro study. Isotonic saline, by contrast, is inert in terms of acid-base chemistry in our model, but causes severe hyperchloraemic acidosis in vivo [17]. Accordingly, it would seem there is an additional beneficial impact of balanced solutions on coronary vascular permeability, beyond pure osmotic and $\mathrm{pH}$ phenomena, most likely related to the ionic composition. The higher concentrations of potassium and free calcium ions would be the most likely ones. In particular, calcium should play an important role in this context, an ion that is not present in sodium chloride solution but in all other solutions tested here.

Both 6 and 10\% hydroxyethyl starch and 4\% gelatine are also based on balanced crystalloid and additionally, contain an artificial colloid. Although previous work suggests that hydroxyethyl starch should be inferior to human albumin in terms of interaction with the glycocalyx to form an endothelial surface layer, the present study applied the artificial colloids in combination with human albumin, thus imitating the scenario of clinical practice. As was to be expected, these preparations were able to maintain vascular barrier function much better than crystalloids [4]. Hypothetically, the mainly negative charges of gelatine, being a protein, could imitate the properties of natural plasma proteins much better than starch, a carbohydrate molecule derived from plants. Indeed, tissue oedema proved to be lower in hearts perfused with gelatine solution, suggestive of a tighter endothelial surface layer.

The in-vitro observations of HP display a direct relationship to the $\mathrm{pH}$ value of the coronary inflow: the more acidosis, the more decrease in LVP and HR. A tight control of acidosis might thus preserve myocardial functioning, which confirms the results of previous studies [17]. It has been shown that acidosis indirectly influences excitation-contraction coupling by decreasing the delivery of calcium to the myofilaments (for example, by inhibition of calcium current at the calcium channel or calcium release of the sacroplasmic reticulum), leading to depressed myocardial performance $[18,19]$.

Additionally, reduction of the calcium concentration in the perfusate due to the addition of artificial solutions (Table 2) might have also affected left ventricular function. In isolated heart models, calcium - one of the key determinants of contractile function - shows a direct relationship with left ventricular developed pressure. It is of note that the $\mathrm{HP}-\mathrm{NaCl}$ group was the only one with an increase in heart rate, possibly due to the reduced potassium concentration. This increase might have offset the effects of reduced $\mathrm{pH}$ and calcium concentration on left ventricular performance.

It is difficult to rule out any direct or indirect effects of the tested artificial colloids on the rate-pressure product. For example, there is a study showing that artificial colloids as additive to cardioplegic solution do have an influence on myocardial oedema development and preserve cardiac function [20]. However, these particular results pertain to ischaemia-reperfusion conditions and there was a different composition of the perfusion solution. Other data suggest that artificial colloids affect endothelial function by reducing endothelium-dependent relaxation and the endothelium-derived hyperpolarising factor [21]. This might interfere with coronary flow regulation, thereby indirectly influencing left ventricular function [22]. However, as we used constant flow perfusion to provide an additional element of constancy in our study, autoregulatory coronary flow mechanisms would be overridden and any such colloidal endothelial effects neutralised. As opposed to such vague possible actions of the different colloids, the changes in $\mathrm{pH}$ of the final perfusate mixtures are closely aligned to the observed functional changes on rate-pressure product (cf. 
Table 2 and Figure 4). There was no such association for the level of potassium.

An important limitation of our results is the use of red blood cell-free perfusates. This is associated with a low viscosity and a low $\mathrm{CaO}_{2}\left(\mathrm{PaO}_{2}\right.$ /oxygen content $)$ and leads to a higher coronary blood flow than in vivo, possibly associated with an increase of shear stress, and consequently an endothelial release of vasoactive substances [23]. Admixture of colloids will also influence viscosity and shear stress versus crystalloid buffers. To alleviate such influences, our perfusion was flow controlled, which additionally eliminated possible effects on flow leading to Gregg's phenomenon [24]. However, because red blood cells influence metabolic and acid-base balances, somewhat different results for the haemodynamic parameters cannot be ruled out in the event of admixture to whole blood [25].

\section{Conclusions}

The presented study investigated the impact of intracoronary perfusion of crystalloidal and colloidal infusion preparations as used in the clinical setting in an isolated guinea-pig heart model. In no case was there evidence for rapid destruction of the endothelial glycocalyx. Nevertheless, the tested solutions produced a notable breakdown of vascular competence, which was most impressive when using isotonic saline and significantly attenuated when using colloidal infusions. Infringement of heart performance showed a correlation with a decreasing $\mathrm{pH}$. Therefore, maintaining the $\mathrm{pH}$ value within a physiological range is important to preserve myocardial function. Prevention of oedema requires the use of colloids prepared in calcium-containing, balanced solutions for volume replacement therapy.

\section{Key points}

- Isotonic saline in colloidal infusions shows notable breakdown of vascular competence.

- It appears important to maintain the $\mathrm{pH}$ value within a physiological range to maintain optimal myocardial contractility.

- Oedema formation may be limited by using colloids prepared in calcium-containing, balanced solutions for volume replacement therapy, instead of crystalloids.

\footnotetext{
Abbreviations

EM: electron microscopy; 6\% HES: 6\% hydroxyethyl starch in Ringer's acetate (Vitafusal 6\%, Serumwerk Bernburg AG, Bernburg, Germany); 10\% HES: 10\% hydroxyethyl starch in Ringer's acetate (Vitafusal 10\%, Serumwerk Bernburg AG, Bernburg, Germany); Gel: 4\% modified Gelatine in ringer's acetate (Gelafusal, Serumwerk Bernburg AG, Bernburg, Germany); HP: heart performance; HR: heart rate; KHB: Krebs-Henseleit buffer; LVP: isovolumetric systolic left ventricular pressure; $\mathrm{NaCl}$ : isotonic saline; PMN: human polymorphonuclear neutrophilic granulocytes; Ri-Ac: Ringer's acetate (RingerAcetat-Lösung Bernburg, Serumwerk Bernburg AG, Bernburg, Germany); RPP: rate-pressure product; TF: transudate formation.
}

\section{Competing interests}

This study was performed using departmental research funding provided by the Bavarian government (Bayerisches Staatsministerium für Wissenschaft, Forschung und Kunst, München; Bavarian State Ministry of Science, Research and the Arts, Munich). In addition, an unrestricted grant was given to the Department of Anaesthesiology, University Hospital Munich by Serumwerk Bernburg AG (Bernburg, Germany). It was not linked to any influence on study design or manuscript approval by the company.

$\mathrm{MJ}$ has held lectures for and received grants from Baxter Deutschland GmbH (Unterschleißheim, Germany), B. Braun Melsungen AG (Melsungen, Germany), Fresenius Kabi Deutschland GmbH (Bad Homburg, Germany), Grifols (Barcelona, Spain), and Serumwerk Bernburg AG (Bernburg, Germany) and is a member of the Grifols Albumin Advisory Board. DC has held lectures for Fresenius Kabi Deutschland GmbH (Bad Homburg, Germany) and has received a research grant from Grifols (Barcelona, Spain). All other authors have no possible conflicts of interest.

\section{Authors' contributions}

YAZ, DC and MJ initiated the study, developed the study protocol, analysed and interpreted the data and wrote the manuscript. BFB, DP, DB and HB performed the experiments, analysed and interpreted the data and coordinated the logistic part of the study. KN accomplished the electron microscopy. BJ performed the statistical analyses. All authors drafted the manuscript and revised it critically for important intellectual content and approved the final manuscript.

\section{Acknowledgements}

The authors are very grateful to Ms U Göttler, Ms S Herzmann, Ms A Sulz, and $\operatorname{Dr} M$ Gruber for expert technical assistance.

\section{Authors' details}

'Department of Anaesthesiology, University Hospital Regensburg, FranzJosef-Strauß-Allee 11, 93053 Regensburg, Germany. 'Department of Anaesthesiology, University Hospital Munich, Nussbaumstr. 20, 80336 Munich, Germany. ${ }^{3}$ Walter-Brendel-Center of Experimental Medicine, University of Munich, Schillerstr. 44, 80336 Munich, Germany.

Received: 2 January 2013 Revised: 29 May 2013

Accepted: 14 September 2013 Published: 14 September 2013

\section{References}

1. Starling E: On the absorption of fluid from the connective tissue spaces. J Physiol (Lond) 1896, 19:312-326.

2. Adamson RH, Lenz JF, Zhang X, Adamson GN, Weinbaum S, Curry FE: Oncotic pressures opposing filtration across non-fenestrated rat microvessels. J Physiol 2004, 557:889-907.

3. Rehm M, Zahler S, Lotsch M, Welsch U, Conzen P, Jacob M, Becker BF: Endothelial glycocalyx as an additional barrier determining extravasation of $6 \%$ hydroxyethyl starch or $5 \%$ albumin solutions in the coronary vascular bed. Anesthesiology 2004, 100:1211-1223.

4. Jacob M, Bruegger D, Rehm M, Stoeckelhuber M, Welsch U, Conzen P, Becker BF: The endothelial glycocalyx affords compatibility of Starling's principle and high cardiac interstitial albumin levels. Cardiovasc Res 2007, 73:575-586.

5. Becker BF, Chappell D, Jacob M: Endothelial glycocalyx and coronary vascular permeability: the fringe benefit. Basic Res Cardiol 2010, 105:687-701.

6. Becker BF, Chappell D, Bruegger D, Annecke T, Jacob M: Therapeutic strategies targeting the endothelial glycocalyx: acute deficits, but great potential. Cardiovasc Res 2010, 87:300-310.

7. Weinbaum S, Tarbell JM, Damiano ER: The structure and function of the endothelial glycocalyx layer. Annu Rev Biomed Eng 2007, 9:121-167.

8. Jacob M, Bruegger D, Rehm M, Welsch U, Conzen P, Becker BF: Contrasting effects of colloid and crystalloid resuscitation fluids on cardiac vascular permeability. Anesthesiology 2006, 104:1223-1231.

9. Chappell D, Jacob M, Hofmann-Kiefer K, Rehm M, Welsch U, Conzen P, Becker BF: Antithrombin reduces shedding of the endothelial glycocalyx following ischaemia/reperfusion. Cardiovasc Res 2009, 83:388-396.

10. Chappell D, Doerfler N, Jacob M, Rehm M, Welsch U, Conzen P, Becker BF: Glycocalyx protection reduces leukocyte adhesion following ischemia/ reperfusion. Shock 2010, 34:133-139. 
11. Jacob M, Paul O, Mehringer L, Chappell D, Rehm M, Welsch U, Kaczmarek I, Conzen P, Becker BF: Albumin augmentation improves condition of guinea pig hearts after $4 \mathrm{hr}$ of cold ischemia. Transplantation 2009, 87:956-965.

12. Zahler S, Kupatt C, Seligmann C, Kowalski C, Becker BF, Gerlach E: Retention of leucocytes in reperfused, isolated hearts does not cause haemodynamically relevant permanent capillary plugging. Pflugers Arch 1997, 433:713-720.

13. Chappell D, Jacob M, Rehm M, Stoeckelhuber M, Welsch U, Conzen P, Becker BF: Heparinase selectively sheds heparan sulphate from the endothelial glycocalyx. Biol Chem 2008, 389:79-82.

14. Zausig YA, Zink W, Keil M, Sinner B, Barwing J, Wiese CH, Graf BM: Lipid emulsion improves recovery from bupivacaine-induced cardiac arrest, but not from ropivacaine- or mepivacaine-induced cardiac arrest. Anesth Analg 2009, 109:1323-1326.

15. Faul F, Erdfelder $E$, Lang AG, Buchner A: G*Power 3: a flexible statistical power analysis program for the social, behavioral, and biomedical sciences. Behav Res Methods 2007, 39:175-191.

16. Shaw AD, Bagshaw SM, Goldstein SL, Scherer LA, Duan M, Schermer CR, Kellum JA: Major complications, mortality, and resource utilization after open abdominal surgery: $0.9 \%$ saline compared to Plasma-Lyte. Ann Surg 2012, 255:821-829.

17. Orchard $\mathrm{CH}$, Kentish JC: Effects of changes of $\mathrm{pH}$ on the contractile function of cardiac muscle. Am J Physiol 1990, 258:C967-C981.

18. Fabiato A, Fabiato F: Effects of $\mathrm{pH}$ on the myofilaments and the sarcoplasmic reticulum of skinned cells from cardiace and skeletal muscles. J Physiol 1978, 276:233-255.

19. Zhou HZ, Malhotra D, Shapiro Jl: Contractile dysfunction during metabolic acidosis: role of impaired energy metabolism. Am J Physiol 1991, 261: H1481-H1486.

20. Coles JA Jr, Sigg DC, laizzo PA: The potential benefits of $1.5 \%$ hetastarch as a cardioplegia additive. Biochem Pharmacol 2005, 69:1553-1558.

21. Dagtekin O, Klisa MA, Gerbershagen HJ, Marcus H, Krep H, Teschendorf P, Fischer $\mathrm{JH}$ : Effects of hydroxyethyl starch, gelatin and dextran on endothelium-derived relaxation in porcine coronary arteries. Pharmacology 2010, 86:267-272

22. Sutherland FJ, Hearse DJ: The isolated blood and perfusion fluid perfused heart. Pharmacol Res 2000, 41:613-627.

23. Koller A, Sun D, Kaley G: Role of shear stress and endothelial prostaglandins in flow- and viscosity-induced dilation of arterioles in vitro. Circ Res 1993, 72:1276-1284.

24. Gregg DE: Relationship between coronary flow and metabolic changes. Cardiology 1971, 56:291-301.

25. Podesser BK, Hallstrom S, Schima H, Huber L, Weisser J, Kroner A, Furst W, Wolner E: The erythrocyte-perfused "working heart" model: hemodynamic and metabolic performance in comparison to crystalloid perfused hearts. J Pharmacol Toxicol Methods 1999, 41:9-15.

doi:10.1186/cc12898

Cite this article as: Zausig et al:: The impact of crystalloidal and colloidal infusion preparations on coronary vascular integrity, interstitial oedema and cardiac performance in isolated hearts. Critical Care 2013 17:R203

\section{Submit your next manuscript to BioMed Central and take full advantage of:}

- Convenient online submission

- Thorough peer review

- No space constraints or color figure charges

- Immediate publication on acceptance

- Inclusion in PubMed, CAS, Scopus and Google Scholar

- Research which is freely available for redistribution 